\title{
Poster: DyMand - An Open-Source Mobile and Wearable System for Assessing Couples' Dyadic Management of Chronic Diseases
}

\author{
George Boateng \\ ETH Zürich
}

\author{
Prabhakaran Santhanam \\ ETH Zürich
}

\author{
Janina Lüscher \\ University of Zürich
}

\author{
Urte Scholz \\ University of Zürich
}

\author{
Tobias Kowatsch \\ ETH Zürich, University of St. \\ Gallen
}

\begin{abstract}
Married adults share illness management with spouses and it involves social support and common dyadic coping (CDC). Social support and CDC have an impact on health behavior and well-being or emotions in couples' dyadic management of diabetes in daily life. Hence, understanding dyadic interactions in-situ in chronic disease management could inform behavioral interventions to help the dyadic management of chronic diseases. It is however not clear how well social support and CDC can be assessed in daily life among couples who are managing chronic diseases. In this ongoing work, we describe the development of DyMand, a novel open-source mobile and wearable system for ambulatory assessment of couples' dyadic management of chronic diseases. Our first prototype is used in the context of diabetes mellitus Type II. Additionally, we briefly describe our experience deploying the prototype in two pre-pilot tests with five subjects and our plans for future deployments.
\end{abstract}

\section{CCS CONCEPTS}

- Human-centered computing $\rightarrow$ Ubiquitous and mobile computing systems and tools; - Applied computing $\rightarrow$ Psychology;

\section{KEYWORDS}

Mobile Computing; Wearable Computing; Mobile Health; Chronic Disease Management; Social Support; Couples

\footnotetext{
Permission to make digital or hard copies of part or all of this work for personal or classroom use is granted without fee provided that copies are not made or distributed for profit or commercial advantage and that copies bear this notice and the full citation on the first page. Copyrights for thirdparty components of this work must be honored. For all other uses, contact the owner/author(s).

MobiCom '19, October 21-25, 2019, Los Cabos, Mexico

(c) 2019 Copyright held by the owner/author(s).

ACM ISBN 978-1-4503-6169-9/19/10.

https://doi.org/10.1145/3300061.3343399
}

ACM Reference format:

George Boateng, Prabhakaran Santhanam, Janina Lüscher, Urte Scholz, and Tobias Kowatsch. 2019. Poster: DyMand - An OpenSource Mobile and Wearable System for Assessing Couples' Dyadic Management of Chronic Diseases. In Proceedings of The 25th Annual International Conference on Mobile Computing and Networking, Los Cabos, Mexico, October 21-25, 2019 (MobiCom '19), 3 pages.

https://doi.org/10.1145/3300061.3343399

\section{INTRODUCTION}

Evidence suggests that for married adults, illness management is mainly shared with their spouses and it involves social support and common dyadic coping (CDC) [9, 11]. Social support and CDC have been shown to have some impact on health behavior and well-being or emotions in couples' dyadic management of chronic diseases such as diabetes in daily life $[4,6,8]$. Hence, understanding dyadic interactions in-situ in chronic disease management could inform behavioral interventions via a digital coach to help the dyadic management of chronic diseases. It is however not clear how well social support and CDC can be assessed in everyday life among couples who are managing chronic diseases such as diabetes mellitus Type II (T2DM). In this work, we describe the development of a novel open-source mobile and wearable system for ambulatory assessment of couples' Dyadic Management of chronic diseases (DyMand). A first prototype is used in the context of T2DM, a common chronic disease affecting $9.4 \%$ of the U.S. population [2] and in Switzerland, $4.9 \%$ of the male Swiss population and $4.2 \%$ of the female Swiss population [10].

\section{DESIGN OF THE ARTIFACT}

The DyMand system as shown in Fig. 1 consists of a smartwatch app ${ }^{1}$, smartphone app ${ }^{2}$ and a cloud-server system

\footnotetext{
${ }^{1}$ https://bitbucket.org/mobilecoach/dymandwatchclient/src/master/ ${ }^{2}$ https://bitbucket.org/mobilecoach/dymand-mobilecoachclient/src/master/
} 


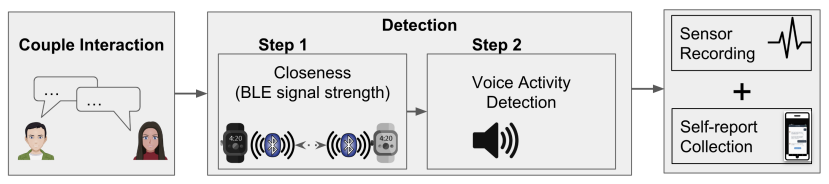

Figure 1: Overview of the DyMand system

built on top of MobileCoach, an open-source software platform for the design of behavioral interventions and ecological momentary assessments [3, 5]. In developing DyMand, experts from the field of computer science, information system and health psychology used justificatory knowledge from prior work $[1,4,6,13]$ about social support, CDC, health behavior and well-being to derive a list of design requirements (DR) that are important for collecting corresponding data in-situ. We describe the requirements below along with our implementation approach.

DR1: Track physical closeness of the couple during waking hours. Implementation: We used the Bluetooth Low Energy (BLE) signal strength of the smartwatch. Each partner is given a smartwatch with one acting as the central and the other acting as the peripheral. The central device continuously scans and tries to find the peripheral device and then logs the BLE signal strength. This data gives an estimate of the physical closeness of the couple throughout the day.

DR2: Collect relevant multimodal sensor data during waking hours every hour when the couple is close and speaking (Fig. 2). Implementation: The smartwatch (Polar M600) collects five minutes of the following sensor data once per hour within the morning and evening hours set by the couples: audio, heart rate, accelerometer, gyroscope, ambient light and BLE signal strength between each partner's smartwatch. The smartphone (Nokia 6.1) collects video, audio and ambient light for three seconds when the subjects are completing the self-report on the phone. We ensure that there are at least 20 minutes between subsequent data collection to reduce the burden of completing the self-reports. To optimize the quality of data collected within that hour, we collect data when the couple is physically close and when the partners are speaking. We use a two-step process. First, we determine closeness using the BLE signal strength between the smartwatches. We check if the signal strength is within a certain threshold, which corresponds to a distance estimate. Then, we determine if the couple is speaking by using a voice activity detection (VAD) algorithm, which is implemented on the smartwatch. The VAD algorithm is a machine-learning algorithm, which was trained to classify speech versus nonspeech. In the case in which this condition of closeness and speaking is not met in the hour, we do a backup recording in the last 15 minutes of the hour.

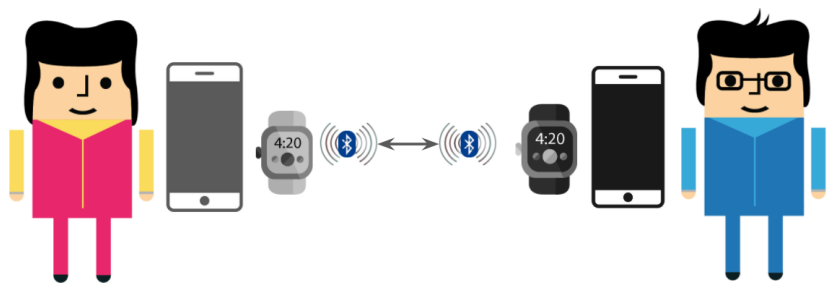

Figure 2: Closeness and Speaking Detection

DR3: Collect self-report data immediately after sensor data collection and at the end of the day (Fig. 3). Implementation: After the five minutes of sensor data collection, the smartwatch app triggers a self-report for each partner to complete on their phones. Additionally, at the end of the day, the self-report is also triggered. The self-report asks questions about social support, CDC, health behavior and emotions.

\section{SIGNIFICANCE TO RESEARCH}

This work seeks to answer the following research question: "How effectively can a mobile and wearable system collect self-report and sensor data about a couple's dyadic interactions in everyday life?" The development of DyMand entails the use of novel approaches such as combining BLE signal strength and VAD to optimize data collection among couples. The method used in developing DyMand can be used by other researchers to develop similar artifacts to collect data to understand various constructs among couples who are managing chronic diseases, and also among other dyadic interactions such as friendships, and sibling and parent-child relationships. Also, another potential research use case is to better understand communication patterns in-situ and performance measures of teams in organizations.

\section{SIGNIFICANCE TO PRACTICE}

The DyMand system results in the collection of subjective data from couples in-situ about social support, CDC and their dyadic management of chronic diseases. Additionally, the system collects objective data such as audio, heart rate, and movements. Audio for example can be used to code constructs such as social support and CDC. Also, audio together with heart rate and movements data can be used to assess emotions, which are an important outcome of the social support and CDC constructs. All the data collected by DyMand can then be used to inform just-in-time-adaptive interventions by for example, a digital coach to improve the management of chronic diseases [7]. 


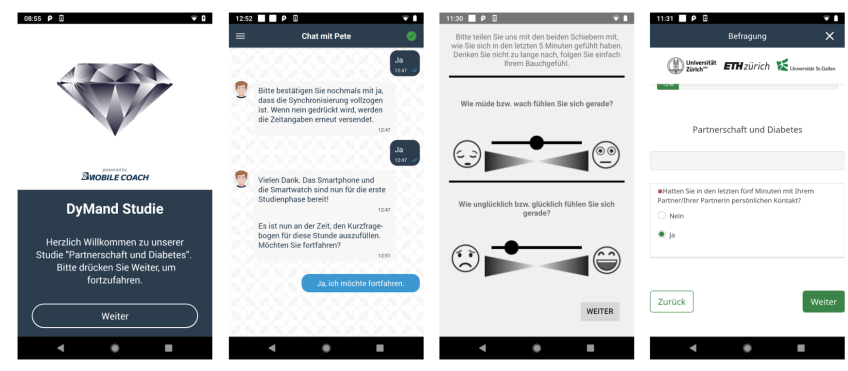

Figure 3: Screenshots of self-report on DyMand app

\section{EVALUATION OF THE ARTIFACT}

Currently, we have run two pre-pilot tests of the DyMand system. We had five subjects in each of the two tests with each test lasting one-week. At the end of the testing, we had collected $900+$ five-minute sensor data along with corresponding triggered self-reports. We had two main technical challenges based on the results of the testing which have been resolved: 1) Unavailability of Internet sometimes resulted in self-reports not being triggered on the phone and 2) A bug in the smartwatch app caused the battery of one of the watches to last for only six hours, which resulted in the watch needing to be recharged during the day and increased the user burden.

We plan to run a study starting in April 2019, funded by the Swiss National Science Foundation [12], through which we will collect various self-report and sensor data in everyday life using the DyMand system. The goal of this study is to understand the relationship between the social support and CDC constructs, and the health behavior and well-being or emotions of couples in which one partner has T2DM. In this study, we aim at including $N=180$ couples ( $n=360$ individuals), where one partner has T2DM. We will collect sensor and self-report data from them for seven days when they spend time together in the mornings and evenings during the weekdays, and the whole day during the weekends.

\section{REFERENCES}

[1] George Boateng and David Kotz. 2016. StressAware: An app for realtime stress monitoring on the amulet wearable platform. In 2016 IEEE MIT Undergraduate Research Technology Conference (URTC). IEEE, 1-4.

[2] Centers for Disease Control and Prevention. 2017. National Diabetes Statistics Report, 2017. (2017). Retrieved July,
2017. from https://www.cdc.gov/diabetes/pdfs/data/statistics/ national-diabetes-statistics-report.pdf

[3] Andreas Filler, Tobias Kowatsch, Severin Haug, Fabian Wahle, Thorsten Staake, and Elgar Fleisch. 2015. MobileCoach: A novel open source platform for the design of evidence-based, scalable and low-cost behavioral health interventions: overview and preliminary evaluation in the public health context. In 2015 Wireless Telecommunications Symposium (WTS). IEEE, 1-6.

[4] Masumi Iida, Mary Ann Parris Stephens, Karen S Rook, Melissa M Franks, and James K Salem. 2010. When the going gets tough, does support get going? Determinants of spousal support provision to type 2 diabetic patients. Personality and Social Psychology Bulletin 36, 6 (2010), 780-791.

[5] Tobias Kowatsch, Dirk Volland, Iris Shih, Dominik Rüegger, Florian Künzler, Filipe Barata, Andreas Filler, Dirk Büchter, Björn Brogle, Katrin Heldt, et al. 2017. Design and evaluation of a mobile chat app for the open source behavioral health intervention platform MobileCoach. In International Conference on Design Science Research in Information System and Technology. Springer, 485-489.

[6] Daisy Miller and J Lynne Brown. 2005. Marital interactions in the process of dietary change for type 2 diabetes. Fournal of Nutrition Education and Behavior 37, 5 (2005), 226-234.

[7] Inbal Nahum-Shani, Shawna N Smith, Bonnie J Spring, Linda M Collins, Katie Witkiewitz, Ambuj Tewari, and Susan A Murphy. 2017. Just-intime adaptive interventions (JITAIs) in mobile health: key components and design principles for ongoing health behavior support. Annals of Behavioral Medicine 52, 6 (2017), 446-462.

[8] Gabriele Prati and Luca Pietrantoni. 2010. The relation of perceived and received social support to mental health among first responders: a meta-analytic review. Journal of Community Psychology 38, 3 (2010), 403-417.

[9] Tuula-Maria Rintala, Pia Jaatinen, Eija Paavilainen, and Päivi ÅstedtKurki. 2013. Interrelation between adult persons with diabetes and their family: a systematic review of the literature. Fournal of family nursing 19, 1 (2013), 3-28.

[10] Schweizerische Diabetes-Gesellschaft. 2015. Diabetes mellitus. Das Wichtigste in KuİLrz. (Feb. 2015). Retrieved September, 2015. from http://www.sdgshop.ch/media/pdf/197-de.pdf

[11] Amber J Seidel, Melissa M Franks, Mary Ann Parris Stephens, and Karen S Rook. 2012. Spouse control and type 2 diabetes management: moderating effects of dyadic expectations for spouse involvement. Family relations 61, 4 (2012), 698-709.

[12] Swiss National Science Foundation. [n. d.]. Measuring the Impact of Social Support and Common Dyadic Coping on Couple's Dyadic Management of Type II Diabetes by a Novel Ambulatory Assessment Application for the Open Source Behavioral Intervention Platform MobileCoach. ([n. d.]). Retrieved January, 2019 from http://p3.snf.ch/ project- 166348

[13] Adela C Timmons, Theodora Chaspari, Sohyun C Han, Laura Perrone, Shrikanth S Narayanan, and Gayla Margolin. 2017. Using multimodal wearable technology to detect conflict among couples. Computer 50, 3 (2017), 50-59. 